\title{
Three Years'Experience of Management of Different Types of Rhinosporidiosis in Rural Part of Western West Bengal
}

\author{
Debdulal Chakraborty, ${ }^{1}$ Chiranjib Das, ${ }^{1}$ Rajesh Hansda ${ }^{1}$
}

\section{Introduction}

\section{$\underline{\text { ABSTRACT }}$}

The incidence of different types of rhinosporidiosis is very high in the rural western part of West Bengal. The treatment of choice is surgical excision and cauterization of the base. The recurrence rate is very high. Aims and objectives

The objectives of this study were to assess the distribution of rhinosporidiosis according to age, sex, presenting features, site of origin, recurrence rate and compare them with literature; and describe the surgical technique to reduce recurrence of the disease.

Materials and methods

This prospective case study was done in the department of Otorhinolaryngology in a tertiary care hospital in the western part of West Bengal from April 2012 to March 2015. Wide local excision of rhinosporidiosis along with electrocautery of the base was done. We took the help of endoscope and microscope whenever needed. Regular follow up with endoscope was done in postoperative period.

$\underline{\text { Results }}$

Out of total 112 patients 62 were male and 50 were female. Commonest age group affected was 2 nd decade. There was no recurrence in patients undergoing operation for the first time. Recurrence was noted in nasopharynx of 3 patients undergoing revision surgery.

\section{Conclusion}

Complete removal of rhinosporidiosis from the base is the basic criterion to reduce recurrence. It is possible by using meticulous technique along with the guidance of endoscope or microscope whenever needed. Regular postoperative follow up with endoscope is must to detect and treat early recurrence.

$\underline{\text { Keywords }}$

Rhinosporidiosis; Otolaryngology; Endoscopy; Epinephrine; Lidocaine; Electrocoagulation

$\mathrm{R}$ hinosporidiosis is a chronic granulomatous disease caused by Rhinosporidium seeberi. ${ }^{1}$ It was first described by Guillermo Seeber in $1900 .^{2}$ It commonly affects nose and nasopharynx. Occasionally conjunctiva, lacrimal sac, maxillary antrum, palate, larynx, trachea, bronchi, urethra and skin are affected. ${ }^{3}$ Disseminated form involves deep viscera and is known as malignant rhinosporidiosis. ${ }^{4}$ It presents as highly

1 - Department of ENT, Bankura Sammilani Medical College, Bankura, West Bengal

\section{Corresponding author:}

Dr Chiranjib Das

email: chirubata.das.87@gmail.com vascular sessile or pedunculated mass. ${ }^{5}$ Most successful treatment is surgical excision and cauterization of the base. $^{6}$ Recurrence is the rule rather than exception. ${ }^{7}$ Dapsone is used by some in recurrent cases. ${ }^{8}$ Though sporadic cases are reported from all over the world, more than $90 \%$ cases are reported from India, Sri Lanka and Pakistan. In India the disease is endemic in coastal parts. ${ }^{9}$ We found a very high incidence of different types of rhinosporidiosis in the western part of West Bengal, which encouraged us to undertake this study.

\section{Aims and objectives}

- To study distribution of rhinosporidiosis according to age, sex, presenting features and compare them 
with previous reports.

- To study distribution of rhinosporidiosis according to site of origin in ear, nose and throat.

- To describe a novel surgical technique to reduce recurrence of the disease.

- To study the recurrence rate and compare it with literature.

\section{Materials and methods}

A prospective case study conducted in the department of Otorhinolaryngology in a tertiary care hospital in the western part of West Bengal from April 2012 to March 2015. A total of 112 cases were studied. A detailed history including age, sex, area of residence, chief complaints and personal habits were recorded. Clinical examination (Fig. 1) and diagnostic nasal endoscopy were done to localize the site of origin and extent of lesion. Recurrent cases and where whole extent of the mass was not visible clinically underwent CT scan of nose and paranasal sinuses to know the exact extent of the mass (Fig. 2). CT scan of neck was done in rhinosporidiosis of oropharynx, laryngopharynx and larynx (Fig. 3). Routine blood investigations such as haemoglobin, total and differential leukocyte count, platelet count, ESR, bleeding time, clotting time, chest X-ray, ECG were done before surgery.

All patients were treated with wide local excision of rhinosporidiosis and electrocautery of the base.

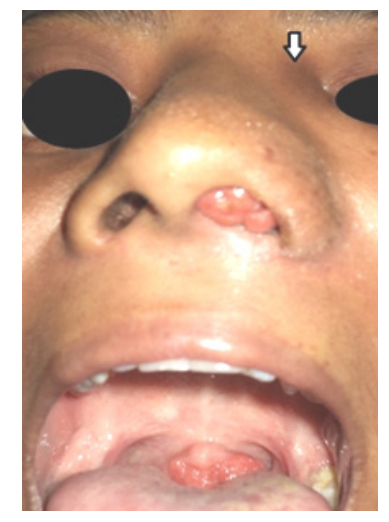

Fig. 1 Rhinosporidiosis seen in left nostril, hanging in oropharynx and swelling of left medial canthal region (white arrow) due to lesion in lacrimal sac

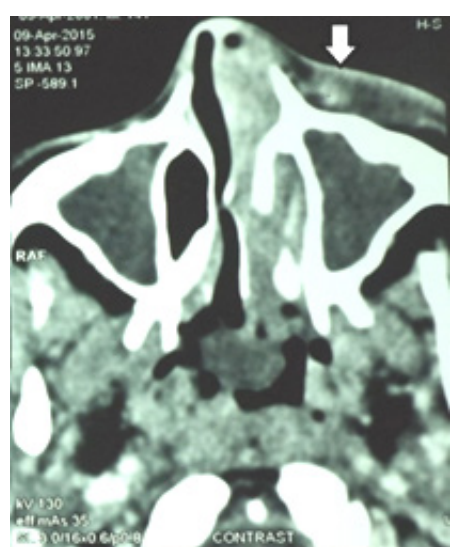

Fig. 2 CECT of nose and PNS axial section showing rhinosporidiosis in left nasal cavity extending to nasopharynx and also to left cheek (white arrow)

Rhinosporidiosis limited to nose was operated under local anaesthesia, except in children. Rhinosporidiosis of nasopharynx, oropharynx and larynx were operated under general anaesthesia. Multiple sites were involved in recurrent cases, which were treated step by step in same sitting.

Nose was prepared with cottonoids soaked with 4\% lidocaine hydrochloride with epinephrine in a ratio of 4:1, 15 minutes prior to surgery. Endoscopic guidance was used where the mass was seen to arise from inferior or middle meatus, posterior part of nasal cavity not visible by headlight and in recurrent cases. About $2 \mathrm{ml}$ of $2 \%$ lidocaine hydrochloride with 1:100000 epinephrine was injected into base of the lesion. The mass was excised

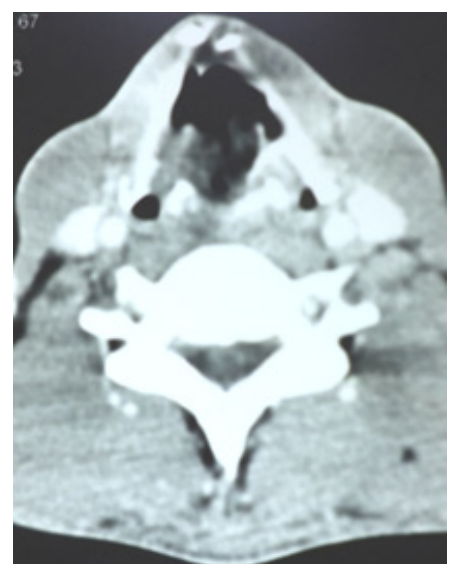

Fig. 3 CECT of neck axial section showing rhinosporidiosis in larynx 
completely from the base. The attachment site and the surrounding mucosa were electocoagulated taking care not to damage the surrounding structures.

Rhinosporidiosis of nasopharynx and oropharynx were removed transorally. With the patient in supine position, neck extended and head flexed, mouth was opened with Boyle-Davis mouth gag with tongue blade (Fig. 4). Four handed technique was used for removal of rhinosporidiosis from nasopharynx. Rubber catheter was introduced through nostril to retract soft palate and uvula. With the help of $70^{\circ}$ endoscope the base of the mass in nasopharynx was visualised. The base was cauterized with a curved bipolar diathermy forceps and the mass was removed per-orally.

Patients with rhinosporidiosis in larynx presented with stridor. Emergency tracheostomy was done. Thereafter with proper investigations, they were put for microlaryngeal surgery. The base cauterized with sucker cum cautery and mass was removed (Fig. 5).

Rhinosporidiosis of lacrimal sac was removed via subciliary incision (Fig. 6).

Excised mass was sent for histopathological study. In recurrent cases, Dapsone $100 \mathrm{mg}$ daily for 6 months was given. Patients were followed up endoscopically at 2 weeks, 1 month, 3 months, 6 months and 1 year.

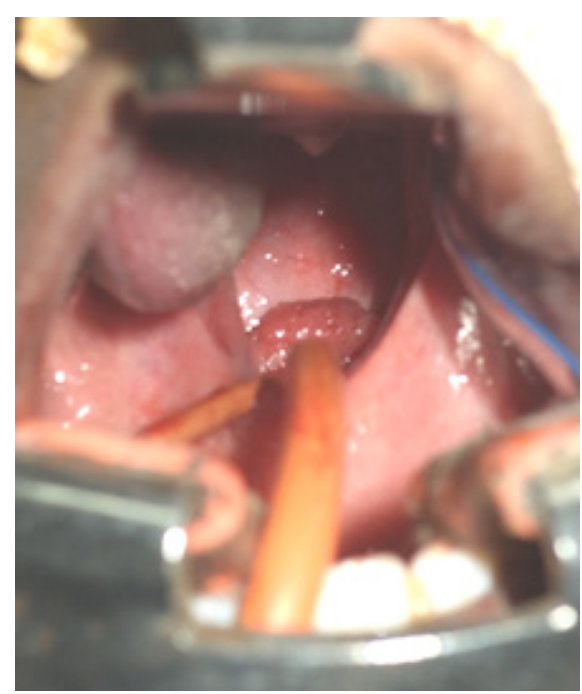

Fig. 4 Transoral removal of nasopharyngeal rhinosporidiosis

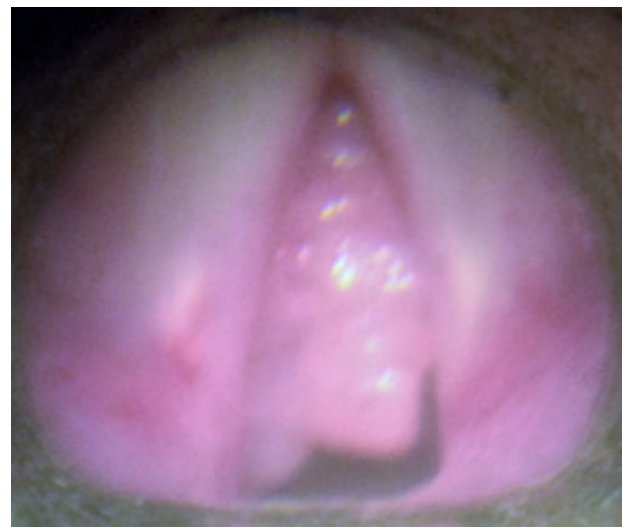

Fig. 5 Rhinosporidiosis in larynx being removed by microlaryngeal surgery

\section{Results}

A total of 112 cases were treated over 3 years from April 2012 to March 2015. There were 62 males (55.36\%) and 50 females (44.64\%) in this study. Patients ranged from 7 years to 77 years of age, being predominantly in the 2nd decade of life (Fig. 7). All of them came from rural area and had history of bathing in pond where cattle were also cleaned. Nasal obstruction $(85.71 \%)$

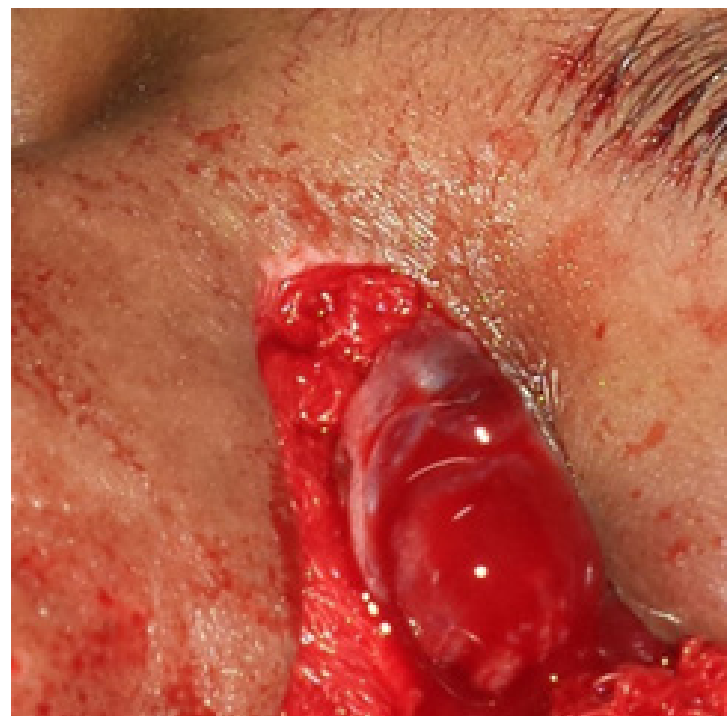

Fig. 6 Rhinosporidiosis of lacrimal sac being removed via subciliary incision 


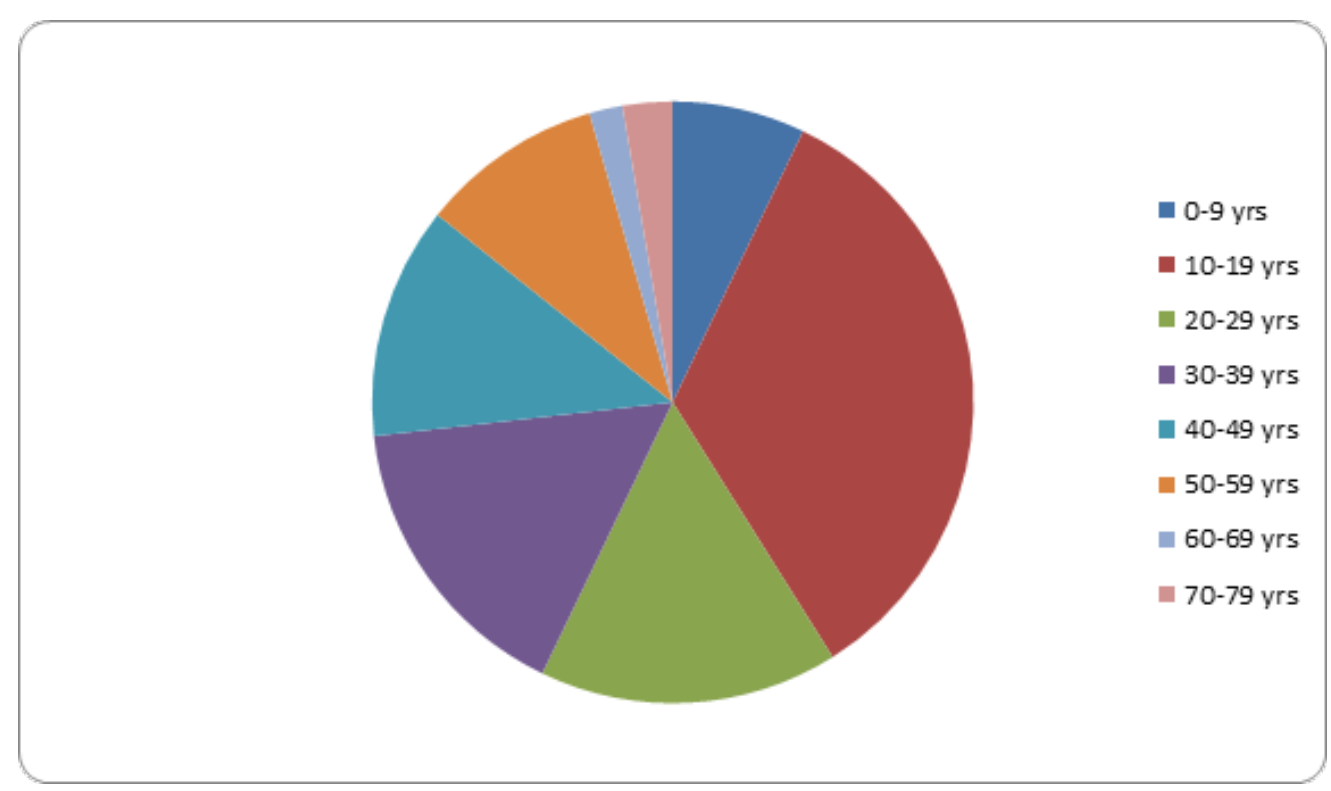

Fig. 7 Distribution of patients according to age

was the most common symptom followed by sensation of something coming out from nose $(78.57 \%)$, nasal discharge $(71.43 \%)$, epistaxis $(32.14 \%)$, change in voice $(9.82 \%)$, foreign body sensation in throat $(6.25 \%)$, difficulty in breathing $(1.79 \%)$ and swelling at medial canthus (0.89\%) (Fig. 8$)$.

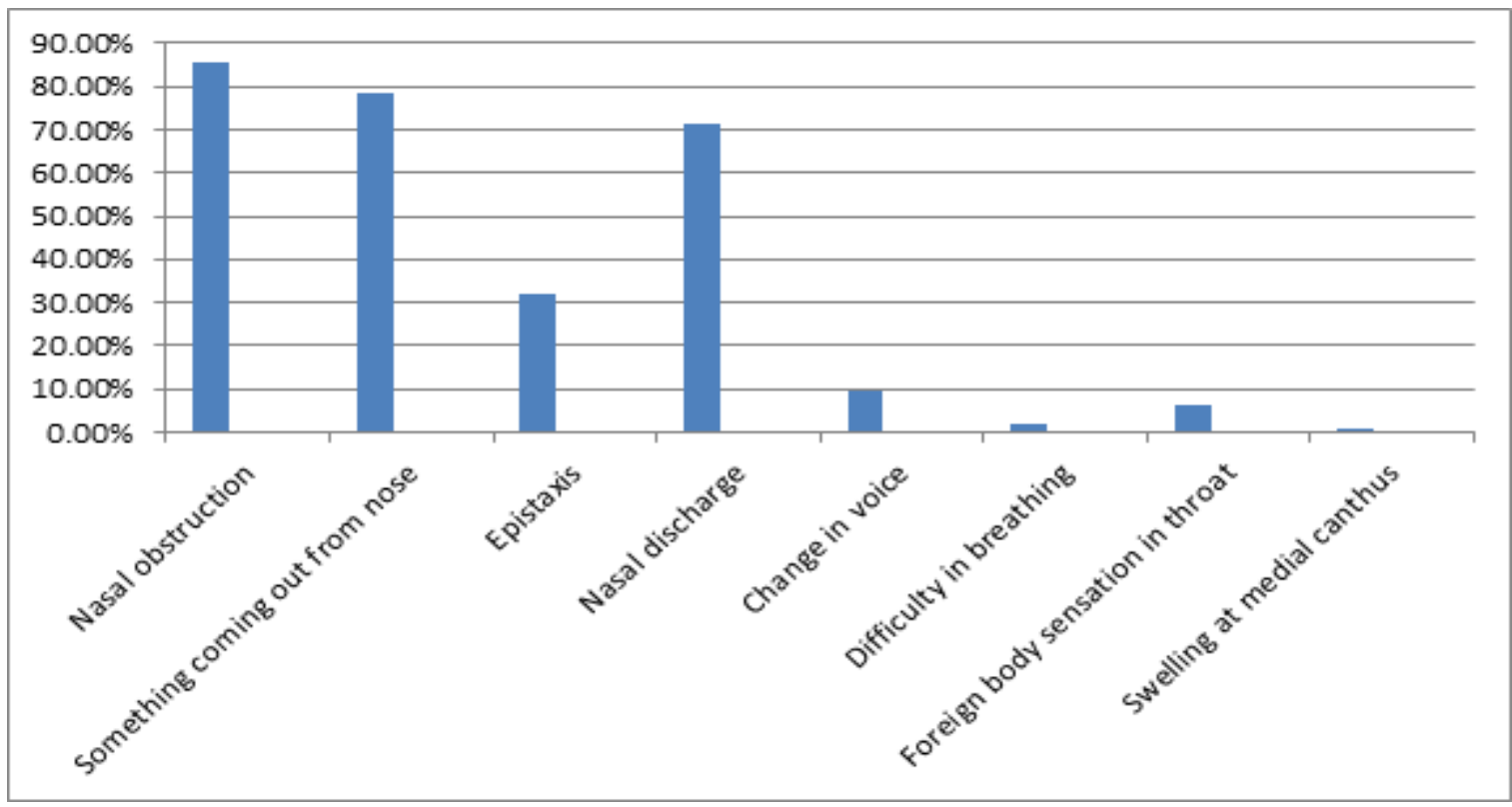

Fig. 8 Distribution of patients according to presenting symptoms 
Nasal septum (52.68\%) was the most common site of origin. Other sites in decreasing order were inferior meatus $(31.25 \%)$, inferior turbinate $(15.18 \%)$, vestibule, floor of the nasal cavity and nasopharynx (7.14\% each), faucial pillars (4.46\%), middle turbinate and soft palate $(3.57 \%$ each), middle meatus $(2.68 \%)$, vallecula, larynx and lacrimal sac $(0.89 \%$ each) (Fig. 9). 11 patients $(9.82 \%)$ had previous history of excision of rhinosporidiosis. All of them had multiple sites of origin. None received Dapsone earlier. All patients were treated with wide local excision and electrocautery of the base.

91 cases were operated under local anaesthesia and 21 cases under general anaesthesia. Severe intra-operative the septum and middle turbinate.

\section{Discussion}

The causative organism was considered as a fungus when Asworth in 1923 described its life cycle establishing the nomenclature Rhinosporidium seeberi. ${ }^{10}$ However, the most accepted hypothesis today is that, Rhinosporidium seeberi belongs to a novel group of fish parasites (Mesomycetozoea) located phylogenitically between fungal and animal divergence. ${ }^{11}$ Stagnant water is suggested as source of infection. Human infection is presumed to occur due to contact of traumatized epithelium with contaminated water. ${ }^{12}$ In our study, all

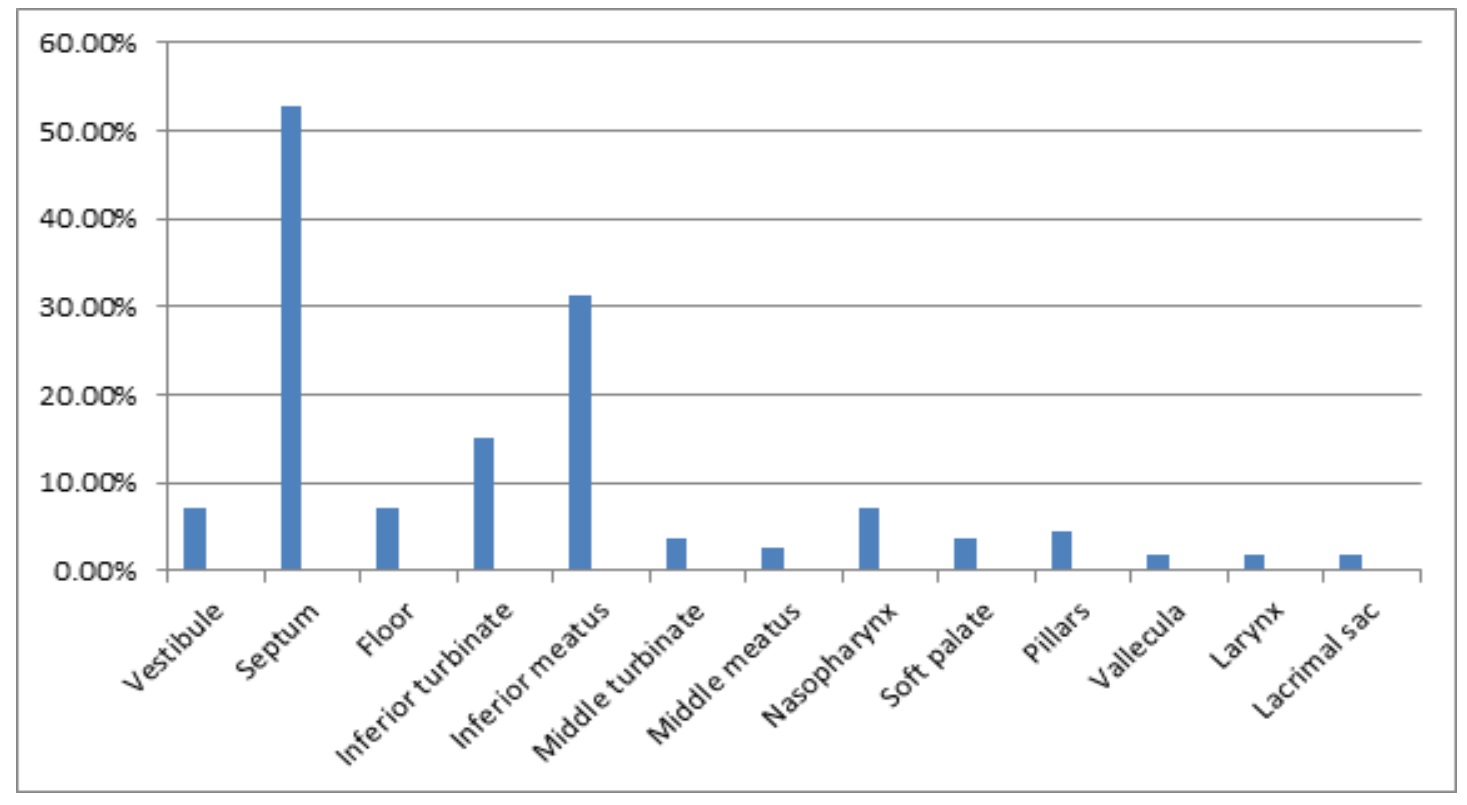

Fig. 9 Distribution of patients according to sites involved

haemorrhage was encountered in 2 cases $(1.79 \%)$ where blood transfusion was needed. Dapsone $100 \mathrm{mg}$ daily for 6 months was given in all recurrent cases. All specimens were reported histopathologically as rhinosporidiosis (Fig. 10). We noticed recurrence or residual mass in 3 patients during the 1 year follow up period, all of which were in the nasopharynx. No recurrence was noted in any other site. Synaechia was noted in 3 cases between the septum and inferior turbinate and in 2 cases between patients came from rural area with habit of pond bathing. Some significant findings emerged while comparing the data of the present series with those of the study reported by Das (1974) from the same district. ${ }^{13}$ Though the commonest age group affected remains the same (2nd decade), the incidence of the disease in female is on the rise. The male: female ratio is now $1.24: 1$ compared to 6.7:1 in the previous report.

The most frequent site is the nasal cavity although 


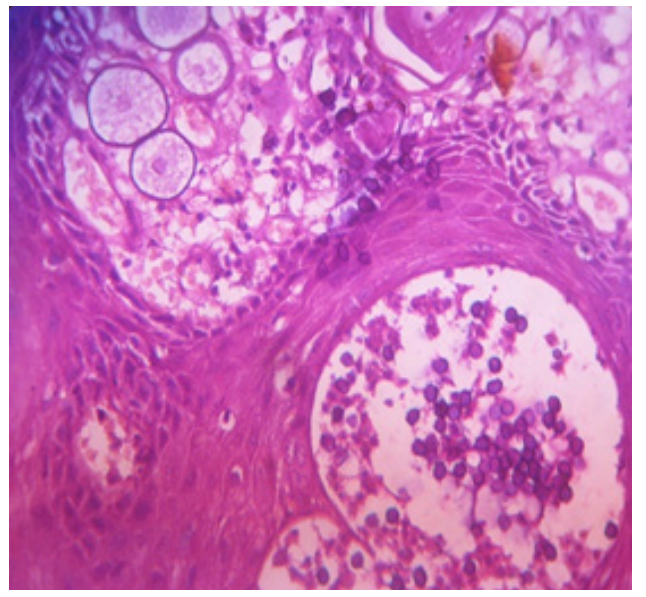

Fig. 10 Photomicrograph showing sporangia in different stages of maturation (100X H\&E)

it is found in other localizations such as the paranasal sinuses, nasopharynx, oral cavity, larynx, conjunctiva, lacrimal sac, urethra, skin, and bone. ${ }^{14}$ Our findings suggest nasal septum (52.68\%) as the most common site of involvement. Our series included 11 patients with previous history of surgery presenting to our hospital with recurrences. All of them had multiple sites of attachment.

The progression of disease is slow and the presenting complaints are nasal blockage, epistaxis, nasal discharge, nasal mass, and sensation of a foreign body. ${ }^{15}$ Clinically rhinosporidiosis presents as a polypoidal lesion, granular, red in colour with multiple yellowish pin headsized spots representing underlying mature sporangia. It is friable, painless, sessile or pedunculated and bleeds on touch. ${ }^{16}$ Our findings revealed nasal obstruction (85.71\%) being the most common presenting symptom.

The treatment of choice is wide local excision and cauterization of the base. Recurrence is the rule rather than the exception. ${ }^{7}$ Most of the recurrences are thought to be due to incomplete removal of mass due to excessive bleeding or auto-inoculation by surgical trauma. ${ }^{17}$ Cottonoids soaked with $4 \%$ lidocaine hydrochloride with epinephrine in a ratio of 4:1 used in rhinosporidiosis of nose ensured adequate decongestion, mucosal anaesthesia, easy access and a bloodless field. Endoscope helps to detect the base of the lesion thereby removing the entire mass under vision. Blood loss also is less due to limited manipulation of the mass and the operative time is shortened. We need to transfuse blood in only 2 patients. All specimens were sent for histopathological examinations which showed sporangia in different periods of maturation and stroma with predominance of plasma cells, lymphocytes and scarce neutrophils.

Literature review suggests a residual or recurrence rate between 10 and $70 \%$. Most of the reports show an incidence on average of $10 \%$, related to incomplete excision of the mass. ${ }^{7}$ In the present study we observed recurrence in 3 cases (2.68\%). All them had recurrence in the nasopharynx. We found that clearance of rhinosporidiosis from nasopharynx is the most difficult job because of multiple attachments and difficulty in exposure and instrumentations. No recurrence was noted in the fresh cases.

Medical treatment is described in the literature. However it is without useful results when used as the only modality of treatment. Dapsone has been used by some authors as an adjuvant to the surgical treatment to reduce the recurrence of disease. It appears to arrest the maturation of the sporangia and promotes fibrosis in the stroma when used as an adjunct to surgery. $8 \mathrm{We}$ used Dapsone in the dose of $100 \mathrm{mg}$ orally per day for 6 months for all 11 patients of revision surgery.

\section{Conclusion}

Rhinosporidiosis is disease of dubious etiology. Although recurrence is very common, in our series it is much less because of meticulous and complete removal. It was possible due to use of epinephrine soaked cottonoids along with the guidance of endoscope or microscope whenever needed. Dapsone can be used as an adjuvant therapy in recurrent cases. The population at risk should be educated to avoid bathing in ponds and rivers open to animals. Regular postoperative follow up with endoscopy is a must to detect and treat early recurrence.

\section{References}

1. Ahluwalia KB. New interpretations in rhinosporidiosis, enigmatic disease of the last nine decades. J Submicros Cytol 
Pathol. 1992; 24:109-14.

2. Karunaratne WA. Rhinosporidiosis in man. London: Athlone Press; 1964. p. 14-8.

3. Sharma KD, Junnarkay RV and Ararwal S. Rhinosporidiosis. J Indian Med Assoc. 1962; 38: 640-642.

4. Kameswaran S, Lakshmanan M. Rhinosporidiosis. In ENT disorders in a tropical environment. Kameswaran S, Kameswaran M, Eds. Chennai, MERF Publications, 1999:1934.

5. Arseculeratne SN. Recent advances in rhinosporidiosis and Rhinosporidium seeberi. Indian J Med Microbiol. 2002; 20:119-31.

6. Ali A, Flieder D, Guiter G, Hoda SA. Rhinosporidiosis: An unusual affliction. Arch Pathol Lab Med. 2001; 125(10):139293.

7. Bhandary S, Natesh V, Chettri S, Kumar A. Rhinosporidiosis. Analysis of cases presenting to a tertiary care hospital in Nepal. Int J Tropical Med. 2012; 8 (1): 1-1.

8. Venkateswaran S, Date A, Job A, Mathan M. light and electron microscopic findings in rhinosporidiosis after dapsone therapy. Trop Med Int Health 1997; 2: 1128-32.

9. Vukovic Z, Bobic-Radovanovic A, Latkovic Z, Radovanovic $Z$. An epidemiological investigation of the first outbreak of rhinosporidiosis in Europe. J Trop Med Hyg. 1995; 98:333-7.
10. Ashworth JH. On Rhinosporidium seeberi with special reference to its sporulation and affinities. Trans Roy Soc Edinburgh 1923; 53:301-42.

11. Herr RA, Ajello L, Taylor JW, Arseculeratne SN, Mendoza L. Phylogenetic analysis of rhinosporidiumseeberi's18S smallsubunit ribosomal DNA groups this pathogen among members of the protoctistan Mesomycetozoa clade. J Clin Microbiol. 1999; 37: 2750-4

12. Karunaratne WAE. The pathology of rhinosporidiosis. J Path \& Bact. 1936; 42:193-202.

13. Das BC. Rhinosporidiosis. Ind J Otolaryngol 1974; 26: 79-84.

14. Makannavar JH, Chavan SS. Rhinosporidiosis: a clinicopthological study of 34 cases. Indian J Pathol Microbiol. 2001; 44: 17-21

15. Weir N. Golding-Wood DG. Infective rhinitis and sinusitis. In: Mackay IS, Bull TR (Eds) Scott-Brown's Otolaryngology Butterworth-Heinemann, 1997; 4 (6): 39-40.

16. Harissi-Dagher M, Robillard N, Corriveau C, Mabon M, Allaire GS. Histopathologically confirmed ocular rhinosporiodiosis in two Canadians. Can J Ophthalmol. 2006; 41:226-9.

17. Das S, Kashyap B, Barua M, Gupta N, Saha R, Vaid L, Banka A. Nasal rhinosporidiosis in humans: new interpretations and a review of the literature of this enigmatic disease. Med Mycol. 2011; 49(3):311-315. 